\title{
COMPARING THE GAP ACCEPTANCE AND TURN TIME PATTERNS OF NOVICE WITH EXPERIENCED DRIVERS FOR TURNS ACROSS TRAFFIC
}

\author{
Eve Mitsopoulos-Rubens, Thomas Triggs \& Michael Regan \\ Monash University Accident Research Centre \\ Melbourne, Victoria, Australia \\ Email: eve.mitsopoulos@muarc.monash.edu.au
}

\begin{abstract}
Summary: Novice drivers are over-represented in crashes involving turns across traffic at intersections and yet little is understood about the contributing factors. The current study proposed to explore the gap acceptance and turn time patterns of novice relative to experienced drivers when turning across traffic. Thirty novice and 30 experienced drivers each carried out a series of trials in a driving simulator that varied in their level of task demand - operationalised in terms of the gap between two successive oncoming vehicles. The novices accepted more gaps than the experienced drivers, although this effect was restricted to gaps falling within the decision "dilemma zone" (higher task demand). Examination of the turn time components for the accepted and, where appropriate, the rejected gaps, revealed that the performance of the novice drivers, in contrast with that of the experienced drivers, was not consistent with the changing task demands. These findings are interpreted in the context of differences between novice and experienced drivers' calibration skill - that is, the ability to match task demands to one's driving capabilities.
\end{abstract}

\section{INTRODUCTION}

Turning across the path of oncoming traffic is considered to be one of the most dangerous and complex manoeuvres in driving (Caird \& Hancock, 2002). The complexity is apparent in the number and the nature of the sub-tasks which drivers must carry out as they plan for, execute, and complete a turn. The decision of whether to turn through a gap in the oncoming traffic stream is time-critical, in that any delays in making or acting upon a decision to accept a given gap could augment the chances of a collision.

Young drivers have been found to be over-represented in crashes which involve turning across traffic (Clarke, Forsyth \& Wright, 2005). While the same is also true for older drivers (e.g. Clarke et al., 2005; Keskinen, Ota \& Katila, 1998), the reasons for young drivers' involvement in such crashes is less clear. One possibility is that young drivers deliberately accept shorter, and therefore, more risky gaps in traffic. However, another possibility is that young drivers have not yet accumulated the sorts of experiences which are needed for facilitating efficient decision making in turn situations involving oncoming traffic. The implication is that, compared with experienced drivers, more decisions are made in the face of uncertainty. Under such circumstances, acceptance of more gaps when turning may be the result of risk taking which is non-volitional in its origin. That there may be a more skill based interpretation of novices' overrepresentation in turning across traffic crashes stems, in part, from Cavallo and Laurent (1988), who found that drivers' accuracy in estimating time to collision information increased with driving experience. 
Research was undertaken which sought to compare, for gap acceptance decisions associated with turning against traffic, the calibration of young novice with experienced drivers. Calibration in driving has been defined as the ability to match the demands of the driving task to one's driving capabilities (Triggs, 1994). The research involved, for each of a group of novice and experienced drivers, matching participants' self-assessed performance with their actual performance on a simulator task, which varied in its level of demand. The relationship between self-assessed and actual performance was then compared across the novice and experienced drivers for evidence of a discrepancy in calibration between them.

Level of demand was operationalised in terms of time gap - that is, the distance, measured in units of time (seconds), between successive oncoming vehicles. Time gaps ranged from short to long with the intention that the shortest and longest time gaps would be associated with relatively lower decision uncertainty (and therefore, task demand) as to whether a gap should be accepted than those time gaps in the middle-size range or the "dilemma zone". It was expected that the novice drivers would show a calibration pattern which differed from that of their experienced driver counterparts. As reported in Mitsopoulos, Triggs and Regan (2006), this expectation was met. A further expectation was that the source of this disconnect between groups would be due to the novices demonstrating, as shown in their actual performance, a reduced appreciation of the task demands relative to the experienced drivers. This would be seen in more liberal gap acceptance in the middle gap size range (higher decision uncertainty) among the novice than the experienced drivers and a pattern regarding the time it took to carry out a turn (turn time) which was independent of the task demand. As all participants were required ultimately to turn, the analysis of turn time patterns pertained to turns made through the gaps of interest (accept cases) and, where relevant, after the gaps of interest had passed (reject cases). The purpose of the current paper is to report on the findings pertaining to the second expectation of the study.

\section{METHOD}

\section{Experimental Design}

A mixed design with two independent variables was employed. The between-subjects variable was driving experience group (novice and experienced) and the within-subjects variable was time gap (3.0, 4.1, 5.2, 6.3 and 7.4 seconds).

\section{Participants}

Sixty drivers took part in the study, with 30 (18 males and 12 females) comprising the novice group (Mean age 19.3 years) and 30 (16 males and 14 females) making up the experienced group (Mean age 41.9 years). The minimum licensing age in Victoria, Australia is 18 years. All novice participants held a Victorian Probationary car driver's licence and had held that licence for no more than six months (Mean 3.1 months). At the time of the study, newly licenced drivers in Victoria, Australia, were required to hold a Probationary (restricted) licence for three years before graduating to a full licence. All experienced group participants held a full (no restrictions) car driver's licence, and had been licenced for a mean of 33.3 years. 


\section{Apparatus}

The Monash University Accident Research Centre (MUARC) driving simulator was used in the current study. For detail, refer to Mitsopoulos-Rubens, Triggs and Regan (2007).

\section{Procedure and Simulator Trials}

All turn events occurred on a two-lane residential road with a single unsignalised " $T$ " intersection and a speed limit of $60 \mathrm{~km} / \mathrm{h}$. As the participant in own-cab drove towards the intersection, a series of four vehicles approached from the opposite direction in the adjacent lane. Participants' task was to turn at the first opportunity where they felt that they had sufficient room in the stream of oncoming traffic to turn safely. At the intersection, the positioning of the oncoming vehicles relative to own-cab was such that participants had two options: either to attempt to turn through the gap between the second and the third vehicles or to wait until all four vehicles had passed the intersection before turning. The time gap variable was the distance in seconds between the front of the second vehicle and the front of the third vehicle.

There were 12 Manual trials, including two practice trials with time gaps of 3.5 and 6.9 seconds, respectively. Each of the five gaps making up the time gap variable was presented twice across the ten experimental trials, which were presented to participants in one of four orders.

\section{RESULTS}

\section{Gap Acceptance}

The first dependent variable to be analysed was gap acceptance: accept, crash and reject. Accept cases were those where the participant decided to turn through the gap and made it through without a collision. Crash cases were those where the participant decided to turn through the gap, but that resulted in a collision. Reject cases were those where the participant decided not to attempt to turn through the gap, instead waiting until all oncoming vehicles had passed the intersection before turning. Figure 1 shows the number of accept, crash and reject cases for each of the novice and experienced groups as a function of time gap. There were too few crash cases to justify their statistical analysis as an independent category. Thus, these data were combined with the reject cases to give a single did not accept category. Also noteworthy from the Figure is that neither driving experience group successfully turned through the 3.0 and 4.1 second time gaps - the smallest time gaps in the series. The final analysis, therefore, comprised all accept and did not accept cases for all time gaps excluding 3.0 and 4.1 seconds.

The final data were analysed using the Generalised Estimating Equation (GEE) method. This statistical approach accommodates for intercorrelation between the outcome variable observations and hence is ideal for application to repeated measures as well as independent groups (Liang \& Zeger, 1986). The exchangeable structure was chosen as the structure of the correlation matrix. As the dependent variable, gap acceptance, represented a binary response (that is, accept/did not accept), the binomial distribution was chosen. The logit link function was selected for modelling the dependent variable, and driving experience group (novice and experienced) and time gap (5.2, 6.3, and 7.4 seconds) served as independent variables. A full 
factorial model was specified in order to explore all main and interaction effects. Non-significant effects were removed from the model one at a time until only significant effects $(p \leq 0.05)$ remained. The final model comprised two significant effects: a main effect of time gap $\left(\chi^{2}(2)=73.62, p=0.00\right)$ and a group by time gap interaction $\left(\chi^{2}(3)=12.47, p=0.01\right)$. The source of the interaction is explored in Table 1.

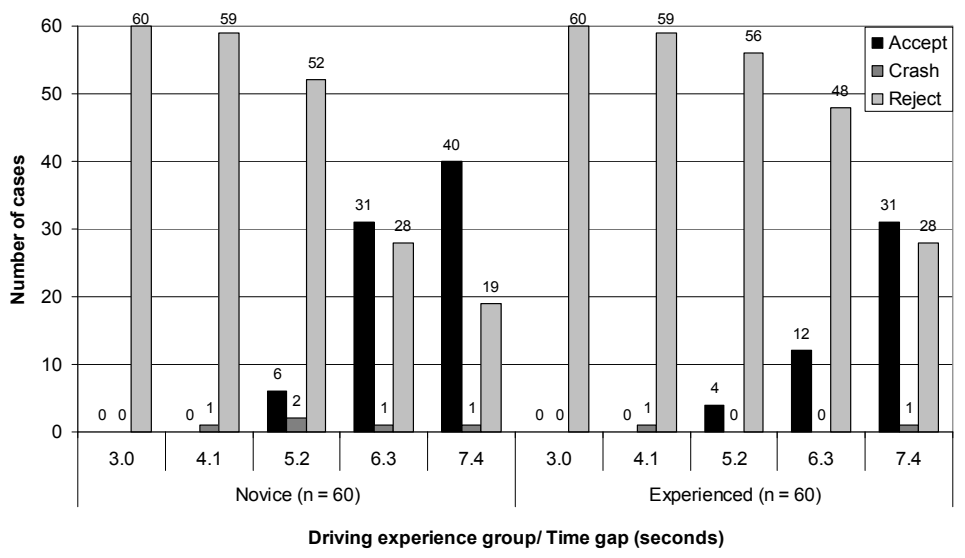

Figure 1. Gap acceptance as a function of driving experience group and time gap

Table 1. Parameter estimates for the driving experience group by time gap interaction

\begin{tabular}{lccccc}
\hline Parameter & B & SE & $\begin{array}{c}\text { Wald } \\
\text { chi-square }\end{array}$ & $\begin{array}{c}\text { Relative odds } \\
\text { ratio }\end{array}$ & $\begin{array}{c}\text { Relative odds ratio 95\% } \\
\text { confidence intervals }\end{array}$ \\
\hline Novice x 5.2 & -2.26 & 0.50 & $20.35^{*}$ & 0.10 & 0.04 to 0.28 \\
Novice x 6.3 & $-3.49 \mathrm{E}-016$ & 0.37 & 0.00 & 1.00 & 0.49 to 2.05 \\
Novice x 7.4 & 0.63 & 0.38 & 2.77 & 1.87 & 0.90 to 3.91 \\
Experienced x 5.2 & -2.71 & 0.51 & $27.58^{*}$ & 0.07 & 0.02 to 0.18 \\
Experienced x 6.3 & -1.45 & 0.32 & $20.28 *$ & 0.23 & 0.12 to 0.44 \\
Experienced x 7.4 (reference) & 0 & & & 1 & \\
\hline
\end{tabular}
$* \mathrm{p} \leq 0.05$

While there was no overall significant main effect of group on gap acceptance, there was a significant interaction between group and time gap. That is, while both the novice and experienced groups demonstrated an increased likelihood of an accept case with an increase in time gap, the pattern of this effect varied between the two groups. For the novices, an increase in the odds of an accept case was found with the increment in time gap from 5.2 to 6.3 seconds. However, no further increase that was statistically significant was observed from 6.3 to 7.4 seconds. For the experienced drivers, no statistically significant increase in the probability of an accept case was found until the increment in time gap from 6.3 to 7.4 seconds. Therefore, at 6.3 seconds, when the novices were beginning to plateau in their probability of an accept case, the performance of the experienced drivers was indicative of a significantly lower probability of an accept decision. At 7.4 seconds, while the novices were still demonstrating a greater likelihood of an accept outcome than the experienced drivers, this difference between groups had narrowed relative to the 6.3 second condition and, so, was no longer statistically significant. 


\section{Turn Time}

For both accept and reject cases, the turn was partitioned into three phases: approach, intermediate, and completion. Each phase was defined with reference to the " 0 " point - the point along own-cab's path when the front of own-cab and the front of the second oncoming vehicle were at their closest while still occupying adjacent lanes. The approach phase constituted the 10 metre distance in the range 15 to 25 metres before the " 0 " point. The intermediate phase represented the 15 metre distance immediately preceding the " 0 " point. The completion phase spanned the distance 10 metres from the " 0 " point.

Figure 2 shows, for the accept and reject cases, respectively, the mean time taken to travel through a phase for each of the novice and experienced groups according to time gap $(5.2,6.3$ and 7.4 seconds) and turn phase. The data for each of the three turn phases were analysed separately, using the GEE method. In every case, the exchangeable structure was selected as the structure of the correlation matrix. The normal distribution and identity link function were chosen for modelling the dependent variable, and a full factorial model was specified. Nonsignificant effects were removed from the model one at a time until only those effects that were significant at $\mathrm{p} \leq 0.05$ remained. The dependent variable was the time taken to travel through a given phase, which, across the three analyses, was the approach phase, the intermediate phase, and the completion phase, respectively.
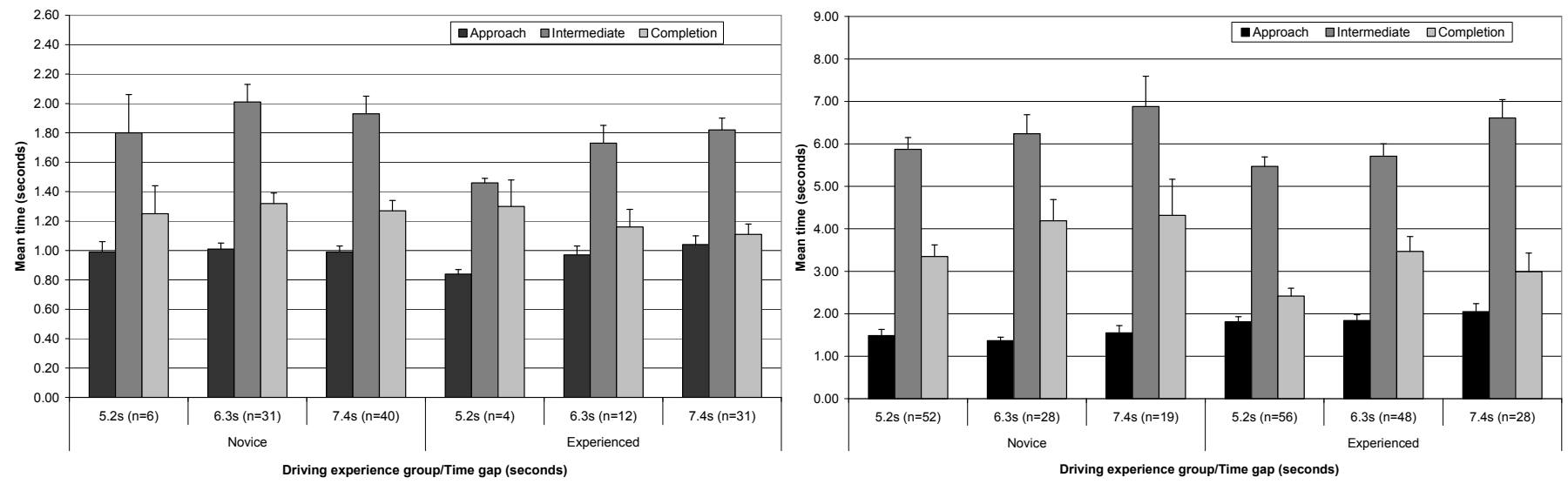

Figure 2. Mean time taken to travel through a phase for each of the novice and experienced groups as a function of time gap and turn phase (Accept cases - left; Reject cases - right)

Approach. Driving experience group (novice and experienced), time gap (5.2, 6.3 and 7.4 seconds), and gap acceptance (accept and reject) served as independent variables in the analysis. The final GEE model comprised only one significant effect: a driving experience group by gap acceptance interaction $\left(\chi^{2}(3)=83.62, p=0.00\right)$. The interaction effect is explored in Table 2 .

Travel time through the approach phase for the drivers in the novice group was significantly shorter than it was for the drivers in the experienced group when the gap was rejected. In contrast, there was no significant difference between the two driving experience groups when the gap was accepted. This discrepancy was driven by the additional finding that, for the experienced drivers, accept cases were associated with a significantly shorter travel time than reject cases. In 
the case of the novice drivers, however, there was no significant difference in travel time between the accept and reject cases. A possible explanation for this pattern of findings is that, by the start of the approach phase, the experienced drivers had already decided whether they were going to accept or reject the gap with which they were presented. Thus, and as reflected in their relatively longer travel time for reject cases, the experienced drivers were already slowing down to wait for all oncoming vehicles to pass through the intersection before continuing their turn through the intersection. The novices, however, travelled through the approach phase in as fast a time when they were accepting the gap as when they were rejecting the gap.

Table 2. Parameter estimates for the driving experience group by turn outcome interaction (approach)

\begin{tabular}{lcccc}
\hline Parameter & B & SE & $\begin{array}{c}\text { Wald } \\
\text { chi-square }\end{array}$ & $\begin{array}{c}95 \% \text { Wald confidence } \\
\text { intervals }\end{array}$ \\
\hline Novice x Accept & -0.81 & 0.10 & $55.62 *$ & -1.02 to -0.60 \\
Novice x Reject & -0.38 & 0.11 & $8.44^{*}$ & -0.64 to -0.12 \\
Experienced x Accept & -0.72 & 0.13 & $57.56^{*}$ & -0.90 to -0.53 \\
Experienced x Reject (reference) & 0 & & & \\
$* \mathrm{p} \leq 0.05$ & & &
\end{tabular}

Intermediate. Driving experience group (novice and experienced) and time gap (5.2, 6.3 and 7.4 seconds) served as independent variables in the analysis. Only accept cases were examined. The final GEE model gave two significant effects: a significant main effect of driving experience group $\left(\chi^{2}(1)=5.13, p=0.03\right)$ and a significant group by time gap interaction $\left(\chi^{2}(4)=18.12\right.$, $\mathrm{p}=0.00)$. The significant interaction is explored in Table 3 .

Table 3. Parameter estimates for the driving experience group $x$ time gap interaction (intermediate)

\begin{tabular}{lcccc}
\hline Parameter & B & SE & $\begin{array}{c}\text { Wald } \\
\text { chi-square }\end{array}$ & $\begin{array}{c}95 \% \text { Wald confidence } \\
\text { intervals }\end{array}$ \\
\hline Novice x 5.2s & 0.05 & 0.26 & 0.03 & -0.46 to 0.56 \\
Novice x 6.3s & 0.21 & 0.15 & 2.09 & -0.08 to 0.50 \\
Novice x 7.4s & 0.11 & 0.14 & 0.59 & -0.17 to 0.39 \\
Experienced x 5.2s & -0.33 & 0.08 & $16.53 *$ & -0.49 to -0.17 \\
Experienced x 6.3s & -0.06 & 0.13 & 0.22 & -0.33 to 0.20 \\
Experienced x 7.4s (reference) & 0 & & &
\end{tabular}

During the intermediate phase, the experienced drivers travelled in significantly shorter time when faced with the 5.2 second gap than when presented with the 7.4 second gap. No significant differences between time gaps were found for the novice group. An explanation for this pattern of findings is that the experienced drivers, who accepted the 5.2 second gap, recognised the elevated risk associated with accepting this particular gap, and so, in an attempt to mitigate the risk, elected to travel through the intermediate phase in shorter time than was the case when the larger gap of 7.4 seconds was accepted. 
Completion. Having accepted a given gap, of interest was whether the novice and experienced drivers differed in the time it took them to travel through the completion phase, and whether this time was influenced by time gap. The independent variables were group (novice and experienced) and time gap (5.2, 6.3 and 7.4 seconds). The GEE showed no significant effects.

\section{CONCLUSIONS}

This paper describes work undertaken as part of a broader program of research on calibration skill in young drivers. The aim of the work reported in this paper was to explore the gap acceptance and turn time patterns of novice relative to experienced drivers. The novices accepted more gaps than the experienced drivers. As expected, this effect was contingent on time gap, with the greater gap acceptance rate of the novices relative to the experienced drivers being significant only at the 6.3 second time gap, which was intended to represent gap sizes in the decision "dilemma zone". Examination of the turn time components revealed that, as expected, the novices were not as adept as the experienced drivers in responding to the changing task demands. The experienced drivers, for example, took less time to travel through the intermediate phase when accepting a 5.2 second gap than when accepting a 7.4 second gap. There were no differences between the two groups during the final, completion phase of the turn. If there were, for example, with the novices travelling in less time following acceptance of the shorter gaps (5.2 and 6.3 seconds), it may have suggested that the novices were responding to the higher task demands later during the turn.

Well developed calibration ability requires more than just an awareness of capabilities and task demands. It involves being able to use that knowledge to know when and how to adjust performance to ensure that the goal of safe driving will be met. To take deliberate risks suggests that the risk taker is aware of the implications of his/her actions. The increased risk acceptance and absence of any compensatory action by the novices in the current study suggests that these behaviours were not deliberate in nature, but a consequence of the less-developed calibration ability of the novice drivers, and more generally, their inexperience. The study outcomes also have important practical implications. For example, through training in calibration in driving, novices will be better equipped to self-regulate their performance in safety critical situations.

\section{ACKNOWLEDGEMENTS}

Support provided under the Australian Postgraduate Award, the Victorian Government Information and Communication Technologies Scholarship scheme, and the MUARC Doctoral Student Research Fund is gratefully acknowledged. Thanks extend to Jason Rubens and Ashley Verdoorn for their programming assistance and Stuart Newstead for his statistical advice.

\section{REFERENCES}

Caird, J.K. \& Hancock, P.A. (2002). Left-turn and gap acceptance crashes. In R.E. Dewar \& P.L. Olson (Eds.), Human factors in traffic safety (pp. 613-652). Tuscon, AZ: Lawyers \& Judges.

Cavallo, V. \& Laurent, M. (1988). Visual information and skill level in time-to-collision estimation. Perception, 17, 623-632. 
Clarke, D.D., Forsyth, R. \& Wright, R. (2005). A statistical profile of road accidents during cross-flow turns. Accident Analysis and Prevention, 37, 721-730.

Keskinen, E., Ota, H. \& Katila, A. (1998). Older drivers fail in intersections: Speed discrepancies between older and younger male drivers. Accident Analysis and Prevention, 30, 323-330.

Liang, K.Y., \& Zeger, S.L. (1986). Longitudinal data analysis using generalized linear models. Biometrika, 73, 13-22.

Mitsopoulos, E., Triggs, T., \& Regan, M. (2006). Examining novice driver calibration through novel use of a driving simulator. Proceedings of SimTect2006. Melbourne, Australia.

Mitsopoulos-Rubens, E., Triggs, T., \& Regan, M. (2007). Examining young novice driver headway choice in a simulated car following task. Proceedings of the 4th International Driving Symposium on Human Factors in Driver Assessment, Training, and Vehicle Design (pp. 558-564). Stevenson, Washington, USA.

Triggs, T.J. (1994). Human performance and driving: The role of simulation in improving young driver safety. Proceedings of the $12^{\text {th }}$ Triennial Congress of the International Ergonomics Association (Vol. 1, pp. 23-26). Toronto, Canada. 